\title{
Aplicación de la estrategia curricular Haramboure como perfec- cionamiento del proceso para el desarrillo de las capacidades físicas generales de los karatecas del Dojo La Pascana de la provincia de Lima
}

Application of curricular strategy Haramboure how improvement of the development of general the physical capacities of the karatecas of the dojo The Pascana of the Lima province

Ray González Haramboure ${ }^{1}$, Sandy Dorian Isla Alcoser ${ }^{2}$

http://dx.doi.org/10.21503/CienciayDesarrollo.2006.v6.08

\section{RESUMEN}

En los últimos años las estrategias con contenidos bioenergéticos y su aplicación al entrenamiento deportivo han adquirido mucha importancia, debido a las investigaciones que se realizan en el mundo y por sus innumerables beneficios en el logro de marcas deportivas, por lo cual justificamos nuestro estudio. El objetivo es contribuir al incremento de las capacidades físicas generales de los karatecas del dojo "La Pascana" de la ciudad de Lima, mediante la aplicación de la estrategia Haramboure. Se evaluó a un solo grupo experimental de 30 deportistas, no queriendo privar a algunos atletas de sus beneficios, con un diseño de pre-post prueba. Procedimos a una evaluación externa del currículum real, encontrando un nivel entre regular y mal. Según los fundamentos teóricos metodológicos de la preparación física, y dentro de la evaluación interna del currículo formal, sólo se observaba el uso del entrenamiento y la omisión de los factores extras. Y dentro de la evaluación interna del currículo real, sólo se utilizaban los del entrenamiento, existiendo la omisión de factores extras. Esta misma deficiencia discrepa con los fundamentos mencionados anteriormente, por la que existía un insuficiente rendimiento deportivo. Entonces, al aplicar la estrategia curricular Haramboure, la cual combina armónicamente todos los contenidos del proceso y coincide con los fundamentos teóricos y metodológicos de la preparación física, se potencializó el rendimiento deportivo.

\section{ABSTRACT}

In the last years the strategies with bioenergetics contents and their application to sport training have acquired much importance, due to the investigations that are made in the world and by its innumerable benefits in the profit of sport marks so that we justify our study. The objective is to contribute to the increase of general physical capacities of karatecas of dojo "the Pascana" in Lima city, by means of the application of the Haramboure strategy. Only one experimental group of 30 sportmen were evaluated, not wanting to deprive to some athletes of these benefits, with a design of pre-post test. We came to an external evaluation of the real curriculum, finding a level between regular and bad. According to the methodologic theoretical foundations of the physical training, and within the internal evaluation of the

1. Catedrático Universidad de la Habana Cuba

2. Catedrático de la Escuela Profesional de Ciencias del Deporte - UAP 
formal curriculo, it was only observed the use of the training and the omission of the extra factors. And within the internal evaluation of real curriculo, we only used the strategies of training existing the omission of extra factors. This same deficiency differs previously with the mentioned foundations, by which an insufficient sport yield existed. Then, when applying the curricular strategy Haramboure, which combines harmonically all the contents of the process and agrees with the theoretical and methodologic foundations of the physical training, we improved the sport yield.

\section{INTRODUCCIÓN}

El proceso de preparación deportiva y el rendimiento alcanzado guardan una estrecha relación de causa y efecto. El primer ejemplo de ello, según Coubertin, P. (1959), es el hecho de que en las primeras ediciones de los Juegos Olímpicos Antiguos, alrededor del año 776 A.C., los vencedores eran casi invariablemente los militares, lo cual se adjudicaba a la rigurosa preparación con que contaban dentro de su formación, que los diferenciaba del resto de los participantes en estas lides. En ese mismo sentido, en la actualidad se reafirma cada día dicha relación, toda vez que se incrementa la norma en las marcas y récords deportivos a medida que se perfecciona progresivamente el proceso de preparación que los competidores reciben.

Dentro de dicho proceso se pondera la preparación física por considerarse el sustento sobre el cual descansa el resto de los tipos de preparación como la técnica, táctica, psicológica y de la preparación física; a su vez, se prioriza el desarrollo de las capacidades como su principal orientación.

Estudios recientes demuestran que varios entrenadores aplican en el proceso de preparación física contenidos de la cultura física oriental, evidenciando logros deportivos de atletas de élite como la del norteamericano Carl Lewis en el deporte del atletismo logrando ser campeón olímpico en 1984, 1988, 1992, 1996; el holandés Inge de Bujin del deporte de natación, logrando ser campeón y recordista olímpico 2000 en 50 y 100 me- tros libre, 100 metros mariposa, y subcampeón en relevo 4X100 libre; el frances Jaques Mayol en el deporte de inmercionismo logrando ser recordista mundial de lastre variable (105 metros) y constante (61 metros).

En la población en estudio, se espera optimizar el proceso de preparación física de los atletas de karate del Dojo La Pascana mediante la aplicación de la estrategia curricular Haramboure, la cual integra una serie de ciencias del deporte de manera armónica y sistémica. Instituciones deportivas en Cuba están aplicando actualmente esta estrategia, como por ejemplo el Laboratorio de Terapias Especiales del ISCF "Manuel Fajardo", tanto para los deportes colectivos como individuales. El resultado ha sido que los atletas ubicados en el grupo experimental superan en rendimiento deportivo al grupo de control.

\section{MATERIAL Y MÉTODO}

Población: Teniendo en cuenta que en el trabajo de investigación es con un solo grupo experimental de 30 atletas, se optó por aplicar a todo el Dojo. Debido a que la Estrategia Curricular Haramboure ya trascendió su fase experimental al haberse constatado su influencia en diversos deportes y países, en lugar de organizar varios grupos comparativos que privara a algunos atletas de sus beneficios, se optó por aplicarla de manera tal que beneficie a todos los atletas involucrados en la investigación. En ese sentido, al aplicar de manera experimental la estrategia a todos los integrantes del Dojo La Pascana y realizar mediciones en diversos momentos para constatar las di- 
ferencias producidas. Basados en la clasificación de Sampieri, R. (1999)

\section{Instrumentos:}

- Silbato

- Cronómetro

- Cinta métrica

- Área de competencia

- Seguimiento longitudinal

\section{PROCEDIMIENTO}

El protocolo de trabajo fue explicado al entrenador y a cada uno de los sujetos en estudio, detallando los contenidos de la estrategia curricular Haramboure la cual podrían influir en su rendimiento deportivo. Se estudió el proceso de preparación física asumiéndolo desde una perspectiva pedagógica considerándolo como el currículo que deben alcanzar los atletas antes de presentarse a las competencias.

Asimismo a cada sujeto se le midió sus capacidades físicas generales, como la fuerza de brazos, fuerza de piernas, fuerza abdominal y la velocidad tanto al inicio como al final, clasificándolos de acuerdo a las siguientes categorías muy bueno, bueno, regular, malo y muy malo. Posteriormente se evaluó la preparación deportiva en su variante formal como real.

Se aplicó la estrategia curricular Haramboure a todos los atletas del Dojo, la cual consta de contenidos de la cultura física oriental al programa de entrenamiento para optimizar así el proceso de preparación deportiva durante un periodo de 3 meses, los días jueves se trabajó fuerza abdominal y velocidad y los sábados fuerza de piernas y fuerza de brazos de 7 a 9 p.m. siendo esos los días de entrenamiento del dojo.
Dentro de la evaluación externa se realizó un análisis utilizando la construcción de pruebas físicas y pruebas estadísticas como el promedio, desviación estándar, significancia de valor, entre otros. También se evaluó de manera interna en este sentido se tuvo en cuenta el criterio tanto del entrenador como de los atletas, de manera tal que respondieran libremente a la pregunta sobre su opinión del trabajo realizado.

\section{RESULTADOS}

De los 30 atletas de karate en estudio, estadísticamente podemos afirmar que:

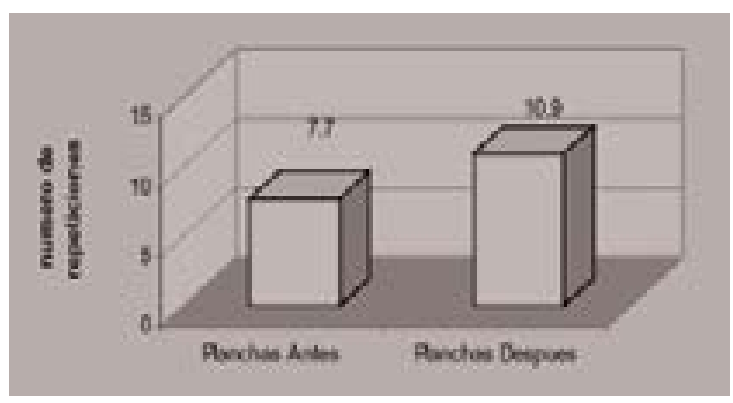

Figura 1. Fuerza de brazos en los atletas de karate - do del Dojo La Pascana

Muestra del rendimiento alcanzado en la anteriormente referida capacidad. Además de esta gran significación biológica, desde el punto de vista estadístico también se constataron diferencias muy significativas.

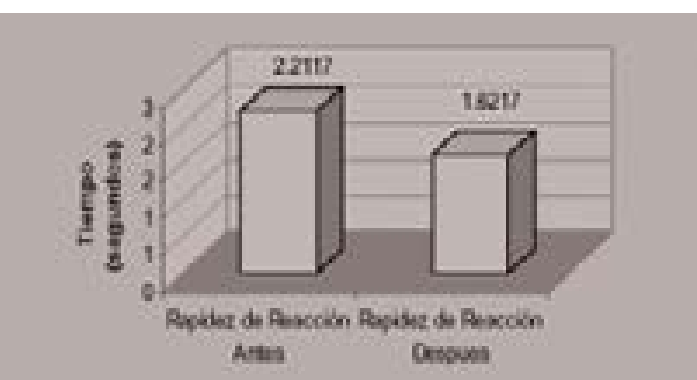

Figura 2. Velocidad de reacción en los atletas de karate - do del Dojo La Pascana

Muestra del rendimiento alcanzado en la anteriormente referida capacidad. Además de esta gran significación biológica, desde el punto de vista estadístico también se constataron diferencias muy significativas. 


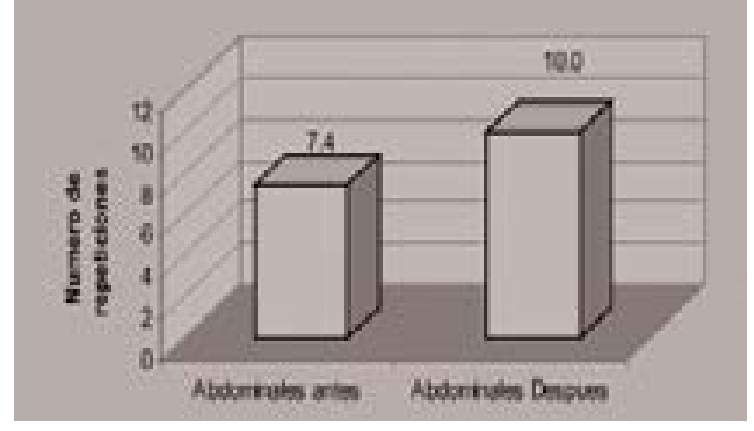

Gráfico 3.Fuerza abdominal en los atletas de karate - do del Dojo La Pascana

Muestra del rendimiento alcanzado en la anteriormente referida capacidad. Además de esta gran significación biológica, desde el punto de vista estadístico también se constataron diferencias muy significativas.

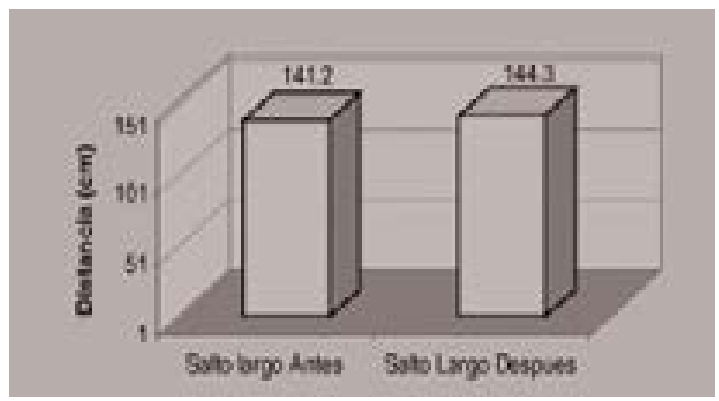

Gráfico 4. Fuerza de piernas - Salto largo en los atletas de karate - do del Dojo La Pascana

Muestra del rendimiento alcanzado en la anteriormente referida capacidad. Además de esta gran significación biológica, desde el punto de vista estadístico también se constataron diferencias muy significativas.

Tabla 1. Comportamiento estadístico del rendimiento alcanzado en las capacidades físicas.

\begin{tabular}{lrrr}
\hline Capacidades Físicas & Promedio & $\begin{array}{c}\text { Desviación } \\
\text { Estándar }\end{array}$ & $\begin{array}{c}\text { Significancia } \\
\text { Valor } p\end{array}$ \\
\hline $\begin{array}{l}\text { Rapidez de Reac- } \\
\text { ción Antes }\end{array}$ & 2,2117 & 2,045 & $\mathrm{p}<0,05$ \\
$\begin{array}{l}\text { Rapidez de Reac- } \\
\text { ción Después }\end{array}$ & 1,6217 & 3,151 & \\
Planchas Antes & 7,7333 & 2,8398 & $\mathrm{p}<0,05$ \\
Planchas Después & 10,9333 & 3,6287 & \\
Salto largo Antes & 141,2333 & 29,4708 & $\mathrm{p}=0,71$ \\
$\begin{array}{l}\text { Salto Largo Después } \\
\text { Abdominales antes }\end{array}$ & 744,2667 & 31,8736 & \\
& & 2,1764 & $\mathrm{p}<0,05$ \\
$\begin{array}{l}\text { Abdominales } \\
\text { Después }\end{array}$ & 10,0333 & 3,2108 & \\
\hline
\end{tabular}

En la Tabla 1, se constata diferencias muy significativas antes y después de la aplicación de la estrategia curricular Haramboure.

\section{DISCUSIÓN}

Uno de los objetivos al iniciar el estudio fue constatar los beneficios que, como consecuencia de la aplicación de la estrategia curricular Haramboure, reciben los atletas. En nuestro medio aún no se publican artículos acerca de estos beneficios, como lo demostró en sus diferentes estudios científicos el Dr. Roberto Haramboure en Cuba, Con respecto a la población con la cual se realizó esta investigación, en aras de perfeccionar el trabajo, se recomienda que el experimento sea repetido teniendo en cuenta tanto el sexo femenino como masculino en atletas de otras provincias, para que sus resultados puedan ser extensivos a todos los karatekas de esta categoría en la provincia.

Debido a que no se encontró antecedentes de aplicaciones experimentales de contenidos de la Cultura Física Oriental para potenciar el funcionamiento orgánico y complementar e intensificar el entrenamiento dirigido al desarrollo de las capacidades físicas de karatekas de la categoría infantil en la provincia, y los resultados obtenidos en este trabajo se corresponden con aquellos alcanzados por otros autores con poblaciones de otras categorías de la provincia de La Habana,

Asimismo, es posible que este tiempo de aplicación de la estrategia curricular Haramboure sea un periodo muy corto para determinar el máximo efecto, tal como sustentan los estudios en otros países. Para tal cometido, sería conveniente realizar además un estudio analizando todo el perfil de la población.

En cuanto al costo de la estrategia curricular Haramboure para el incremento del rendimiento deportivo, se comprobó que la aplicación es muy económica y está al alcance de todos los estratos sociales, teniendo una serie de capacita- 
ciones en la reestructuración el programa deportivo y aplicaciones prácticas de algunos factores extras en el entrenamiento deportivo.

\section{CONCLUSIONES}

La aplicación de la estrategia curricular Haramboure incrementa el rendimiento deportivo y en especial la preparación física como base de las demás preparaciones.

Se ha logrado incrementar el porcentaje de desarrollo en las capacidades físicas de estos atletas no solo de una muy alta significación tanto biológica como estadística, sino que esos valores nunca antes habían podido ser alcanzados con el proceso habitualmente utilizado.

Los beneficios alcanzados, debido al modo en que se aplicó la estrategia, se obtuvieron con un menor tiempo hábil de trabajo que el usualmente destinado al entrenamiento deportivo.

La aplicación de esta estrategia curricular es una alternativa para el incremento del rendimiento deportivo tanto en deportes colectivos como individuales.

\section{REFERENCIAS BIBLIOGRÁFICAS}

- Egami Shigeru. The heart of karate do. Ed. Kodansha LTD, Tokyo 1986.

- Forteza de la Rosa, A Ranzola Rivas, A Bases metodológicas del entrenamiento deportivo. Ed. Científico técnica, Ciudad de La Habana, 1986.

- González Roberto. Propuesta del programa Haramboure para complementar la preparación de los atletas de la Selección Juvenil de Karate do de Ciudad de La Habana. Instituto Superior de Cultura Física. Ciudad de La Habana. 2000. Tesis doctoral.
- Kido, el camino del espíritu. Ciudad de La Habana, 1999.

- Entrenamiento energético ¿El doping del nuevo milenio?. Ciudad de La Habana, 2000.

- El Zen: De Shaolin al Olimpismo. Ciudad de La Habana, 2001.

- Programa de preparación para los karatekas cubanos del estilo Goju ryu, Ciudad de La Habana 2000.

- Grosser, M y Muller, H. Power Stretch. Ed. Hispano europea. España, 1989.

- Hahn, E. Entrenamiento con niños y jóvenes. Ed. Martinez Roca, S,A Barcelona, 1998.

- Harre, D. Teoría y metodología del entrenamiento deportivo moderno. Sportverlag, Berlín, 1973.

- Higaonna, M. Karate do tradicional Ed. Paidotribo, Barcelona, 1996.

- Jwing Ming, Y. La técnica oriental para mejorar la salud y las Artes Marciales. Ed. Arbol. México. 1992.

- Kadowaky T. Shiatsu masaje japonés en México. Instituto médico Tao. México A.C 1990.

- Kanazawa H. Dynamic power of karate. Dragoon books publications. Tokio, 1986. Laboratorio de Terapias Especiales. Documentos de archivo. Instituto Superior de Cultura Física. Ciudad de La Habana. Cuba. 2000.

- Matvéev. L. P. Fundamentos del entrenamiento deportivo. Ed. Ráduga. Moscú, 1983.

- Organización Mundial de la Salud (OMS). Reunión de Alma Atá, 1978. 
- Ozolin, N. G. Teoría y metodología del entrenamiento deportivo. Ed. Progreso, Moscú, 1970.

- Posner J, G. Análisis de currículo. Segunda edición. D’Vinni Editorial Ltda, Colombia, 1998.

- Prats, J Ma. Qigong, el arte secreto chino de la respiración para la salud física y mental. Ed. Alas, Madrid, 1985

- Rodríguez Velásquez, Jaime Ricardo Dr., Diccionario Enciclopédico de Ciencias y Técnicas Deportivas. Ediune, Lima, 1999.

- Solís Femández L. La competición de Karate. Ed. Paidotribo, Barcelona 1994.
- Tamano, Toshio. Curso de karate, el karate Gojuryu. Ed. De Vecchi, S.A. Barcelona, 1992.

- Toguchi, Seikichi. Okinawan Gojuryu, Fundamentals of Shoreikan karate. Ohara Publications Inc. California 1976.

- Tung Kuo Tsao. El Qigong y las artes marciales. Barcelona, 1990

- Yves Requera. Qigong, gimnasia china para la salud y la longevidad. Ed. Liebre de Marzo, Barcelona, 1993. 